\title{
Measurement, Modeling and Improvement of Optical Image Quality in Human Eyes
}

\author{
R. NAVARRO* \\ Instituto de Optica "Daza de Valdés", Consejo Superior de Investigaciones \\ Científicas (CSIC), Serrano 121, 28006 Madrid, Spain
}

\begin{abstract}
New objective methods to measure the optical aberrations of the eye are reviewed, in particular the probably more representative ones: Hartmann-Shack wavefront sensor and laser ray tracing. They are shown to be robust and provide highly reliable data, which are permitting to obtain many new results about the optics of the eye in basic and clinical studies. In addition, different experiments have demonstrated the correction of eye's aberration. Again two representative approaches are reviewed. The first results were obtained with the close-loop adaptive optics system developed at the University of Rochester. Later on, phase plates made by photosculpture in photoresist (placed in front of the eye like conventional lenses) have also permitted the correction of ocular aberrations. This is a new, but already very active field of research, which has open many new questions and a wide variety of applications.
\end{abstract}

PACS numbers: 42.66.- $\mathrm{p}, 42.15 . \mathrm{Dp}$

\section{Introduction}

In the last years, there has been an increasing interest on the optics of the eye. On the one hand, the new refractive surgery techniques are not only demanding a precise knowledge of the optical system of the eye that is going to be modified, but they need means to predict the final result. Refractive surgery is producing an enormous impact on public visual health and economy. On the other hand, a technological revolution has occurred in parallel. Many new technologies developed for optical microscopy, communications or astronomy, as well as devices such as lasers, scanners, CCD cameras, computers, optical design software, etc., have been

*e-mail: r.navarro@io.csic.es; URL: http://www.io.csic.es/rafael.htm 
applied to physiological optics, which has produced a significant step ahead in our knowledge of the optics of the eye. In particular there has been a renewed effort in the development of techniques for estimating the wave aberration of the human eye [1-5] partly stimulated by the interest in assessing the changes in optical quality produced by refractive surgery [6-8] and by the desire to compensate the ocular aberrations to achieve diffraction-limited optics $[9,10]$. In theory, it would be possible not only to compensate aberrations with external aids, but even to correct them permanently by surgery or other techniques.

Subjective methods to measure aberrations (astigmatism, spherical, chromatic, etc.) of the eye have been developed and used since a long time ago. In 1961 Smirnov [11] reported the first measurements of the overall wave aberration in the human eye, obtained by means of a spatially resolved subjective refractometer. In that publication he already suggested that "in principle, it is possible to manufacture a lens compensating the wave aberration of the eye in the complex form of plates of errors". Nevertheless, subjective measurements have important drawbacks (time consuming, reliability may depend on subject, etc.) and limitations (our poor peripheral visual performance precludes off-axis measurements). In recent years, new available technologies made possible to develop objective methods, such as the objective version of the Howland aberroscope [12], Hartmann-Shack wavefront sensor (H-S) [2], laser ray tracing (LRT) [3] and Tcherning type aberroscope [4]. Different studies [13] have demonstrated the high reliability and robustness of some of these methods. Among the above experimental methods, here we shall overview two of them, $\mathrm{H}-\mathrm{S}$ and LRT, which are probably the more representative ones.

Once we can measure ocular aberrations, the next challenge is to correct them. The first successful experiments were carried out using a closed-loop adaptive optics device where aberrations were measured by a $\mathrm{H}-\mathrm{S}$ sensor and corrected by means of a deformable mirror [9]. These sophisticated adaptive optics systems are, however, far from the idea of a "special single lens" and it is hard to imagine them working outside the environment of the optical laboratory or clinic. In this sense, we intended to verify Smirnov's suggestion experimentally, by manufacturing and testing "special lenses", which, in our approach, consisted of phase plates adapted to compensate optical aberrations in individual eyes [10]. The variety of applications of aberration measurement and correction is potentially enormous, because it could allow to improve vision, even beyond normal limits [9], and also improve resolution in fundus clinical exams or surgery.

\section{Measurement of ocular aberrations}

The different methods, available so far, are based on measuring geometrical or ray aberrations and then obtaining the wavefront error by numerical integration. As shown in Fig. 1, rays are normal to the wavefront. The geometrical aberration 


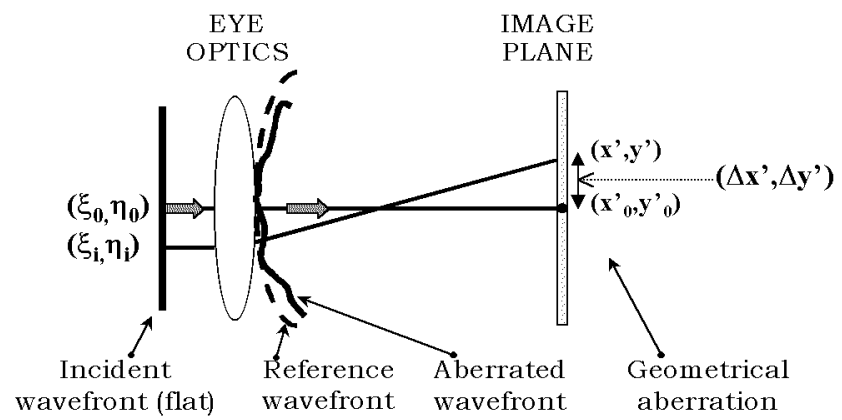

Fig. 1. Ray and wave aberration. Rays $\perp$ wavefront.

of a ray passing through a given pupil position $\left(\xi_{i}, \eta_{i}\right)$ is defined (see Eq. (1)) as the geometrical displacement between the location where it intersects the image plane $\left(X^{\prime}\left(\xi_{i}, \eta_{i}\right), Y^{\prime}\left(\xi_{i}, \eta_{i}\right)\right)$ and a reference ideal location $\left(X_{0}^{\prime}, Y_{0}^{\prime}\right)$. In other words, in an aberration-free optical system, each spot would lie on its reference ideal position at the image surface, $X^{\prime}=X_{0}^{\prime} ; Y^{\prime}=Y_{0}^{\prime}, \forall i$.

In the presence of aberrations, the spots are displaced from their reference positions; the geometrical aberration is defined simply by the magnitude of these displacements:

$$
\Delta x^{\prime}\left(\xi_{i}, \eta_{i}\right)=\frac{X^{\prime}\left(\xi_{i}, \eta_{i}\right)-X_{0}^{\prime}\left(\xi_{i}, \eta_{i}\right)}{f^{\prime}}, \Delta y^{\prime}\left(\xi_{i}, \eta_{i}\right)=\frac{Y^{\prime}\left(\xi_{i}, \eta_{i}\right)-Y_{0}^{\prime}\left(\xi_{i}, \eta_{i}\right)}{f^{\prime}} .
$$

In these equations, the displacements are given in dimensionless tangent units, by dividing by the focal length of the lens used for imaging the spots. This is especially relevant for double-pass measurements in the human eye, where we do not have access to the retinal image plane, and thus we cannot measure length units but only tangents or angles. For a given point object with fixed coordinates $\left(X_{0}, Y_{0}, Z_{0}\right)$, we can plot the aberrations $\left(\Delta x_{i}^{\prime}, \Delta y_{i}^{\prime}\right)$ of all the rays delivered, passing through different pupil positions, to obtain a spot diagram representing the geometrical aberrations of each sample (pencil of light) of the beam passing through the optical system.

There is a close relationship between the ray aberration and the slope (or first derivative) of the wavefront at a given pupil location. The components $\left(\Delta x_{i}^{\prime}, \Delta y_{i}^{\prime}\right)$ of the ray aberration are proportional to the slopes (tangents) of the wave aberration $W(\bar{\xi}, \bar{\eta})$ in both axes, where $\bar{\xi}=\xi / R_{\mathrm{p}}, \bar{\eta}=\eta / \mathrm{R}_{\mathrm{p}}$ are dimensionless canonical pupil coordinates and $R_{\mathrm{p}}$ is the pupil radius:

$$
\Delta x^{\prime}=\frac{1}{R_{\mathrm{p}}} \frac{\partial W(\bar{\xi}, \bar{\eta})}{\partial \bar{\xi}}, \quad \Delta y^{\prime}=\frac{1}{R_{\mathrm{p}}} \frac{\partial W(\bar{\xi}, \bar{\eta})}{\partial \bar{\xi}} .
$$

We can estimate $W$ by integrating Eq. (2). However, instead of a direct integration that is numerically unstable, it is better to consider an expansion of the 
wave aberration in terms of Zernike polynomials. Zernike polynomials are well suited for describing the wave aberration, because they form a complete orthogonal basis to represent a function defined over a circular support with a unit radius [14]. Moreover, orthogonality implies that the total value of the wave aberration variance can be found by adding the individual contributions of each Zernike term. It is common to consider a 7 th order approximation, that is 35 terms, under the assumption that higher orders will be very small so that we can have them cut-off

$$
W(\bar{\xi}, \bar{\eta}) \approx \sum_{k=1}^{35} Z_{k} \cdot P_{k}(\bar{\xi}, \bar{\eta})
$$

where $Z_{k}$ are the coefficients of the expansion, in microns, $(\bar{\xi}, \bar{\eta})$ are canonical pupil coordinates, and $P_{k}$ are dimensionless Zernike polynomials. To estimate $W$ from the raw data (ray aberrations), we only have to substitute $W$ by its expansion (Eq. (3)) in Eq. (2). Then, instead of numerical integration of the raw data, we can compute the partial derivatives of Zernike expansion of $W$. Provided that the number of rays is greater than 35 , we have an overdetermined linear system of equations, where the $Z_{i}$ are the unknowns, so that it can be solved by a standard least-square fitting.

\subsection{Hartmann-Shack wavefront sensor}

The operating principle of the $\mathrm{H}-\mathrm{S}$ wavefront sensor is well known, and its application to the human eye is becoming popular. In the $\mathrm{H}-\mathrm{S}$ wavefront sensor, a monolithic microlens array, located in a plane conjugated to the exit pupil, samples the wavefront in parallel. Each lenslet selects a light pencil (whose diameter is that of the lenslet) and forms a spot of light at its focal plane. For an aberration-free

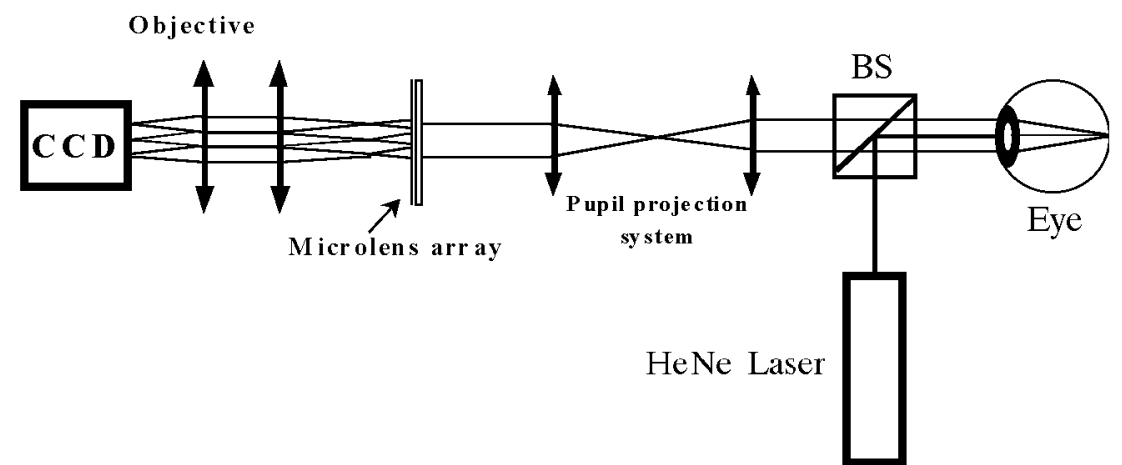

Fig. 2. Hartmann-Shack wavefront sensor. The microlens array samples the wavefront forming an array of spots. BS - beam splitter. 
system, if the object is at the focal point, then the emerging wavefront is flat and thus, the array of image spots at the microlenses focal plane has a regular spatial distribution identical to that of the microlens themselves (square, hexagonal, etc.). In the presence of aberrations, the light pencils passing through the microlenses are tilted relative to the corresponding aberration-free ones, and thus, the image spots are shifted from their ideal or reference position. The tilt is simply the average slope of the wavefront across the microlens pupil.

Figure 2 shows the $\mathrm{H}-\mathrm{S}$ system built in our laboratory [13]. The light from a HeNe laser is reflected by a beam splitter, enters the eye through the center of the pupil and forms a Gaussian spot on the retina. The light reflected passes again through the pupil and a pair of lenses, which project the image of the pupil onto a microlens array. The array of images (spots) provided by the microlenses is focused on a CCD camera to be digitally recorded and analyzed.

\subsection{Laser ray tracing}

Laser ray tracing (LRT) consists of delivering, sequentially, a bundle of light pencils (unexpanded laser beams) coming from the same point object, but passing through different locations at the eye's pupil. The trajectories of the light pencils (rays) are controlled by means of a two-dimensional XY laser scanner plus an additional collimator (see Fig. 3). The spot formed by each ray on the retina,

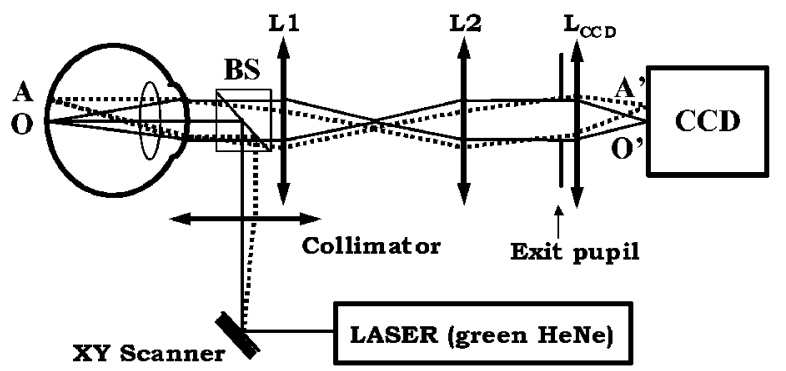

Fig. 3. Laser ray tracing system. A two-dimensional scanner deflects the laser pencil which after collimation, enters the eye through the desired pupil position. The spot formed by each ray (laser pencil) onto the retina is imaged on a CCD camera.

is imaged onto a CCD camera. Both the scanner and the camera are controlled and synchronized by means of a PC, which is also used to analyze each image, computing the centroid of each spot. Although LRT is sequential, it is possible to deliver rays and to record the associated images reasonably fast. In the current version of the system we can trace and record 8-9 rays/second.

\section{Aberrations in normal and clinical eyes}

Thanks to these objective methods, the amount of experimental data available is growing very fast, and some recent publications report results over large 
populations [15]. It appears to be a random variation in the eye's aberrations from subject to subject. Roughly speaking, after canceling second order aberrations (defocus and astigmatism) with ophthalmic lenses, the RMS wavefront error for a $6 \mathrm{~mm}$ pupil is of the order of $\lambda$ (about half a micron), that is very far from the diffraction limit $(\lambda / 14)$. Nevertheless there is a large variation between individuals, and the optical quality can depart substantially from $\lambda$.

Aberrations also change across visual field within an eye [16], as it is illustrated in Fig. 4. The wave aberration increases gradually, and roughly linearly with eccentricity, so that at $40^{\circ}$ the RMS wavefront error is roughly double than the RMS value on the visual axis $\left(0^{\circ}\right)$. Compared to a conventional optical system, the human eye shows a poor optical quality on-axis, very far from the diffraction limit. (RMS wavefront error, after canceling second order aberrations, is about $1 \lambda$ for a $6 \mathrm{~mm}$ pupil). However, these results show how the optical (aberrations) and image (modulation transfer function - MTF) quality declines slowly and gradually with retinal eccentricity. Such decline of optical performance with retinal eccentricity is much slower and gradual than previously thought.

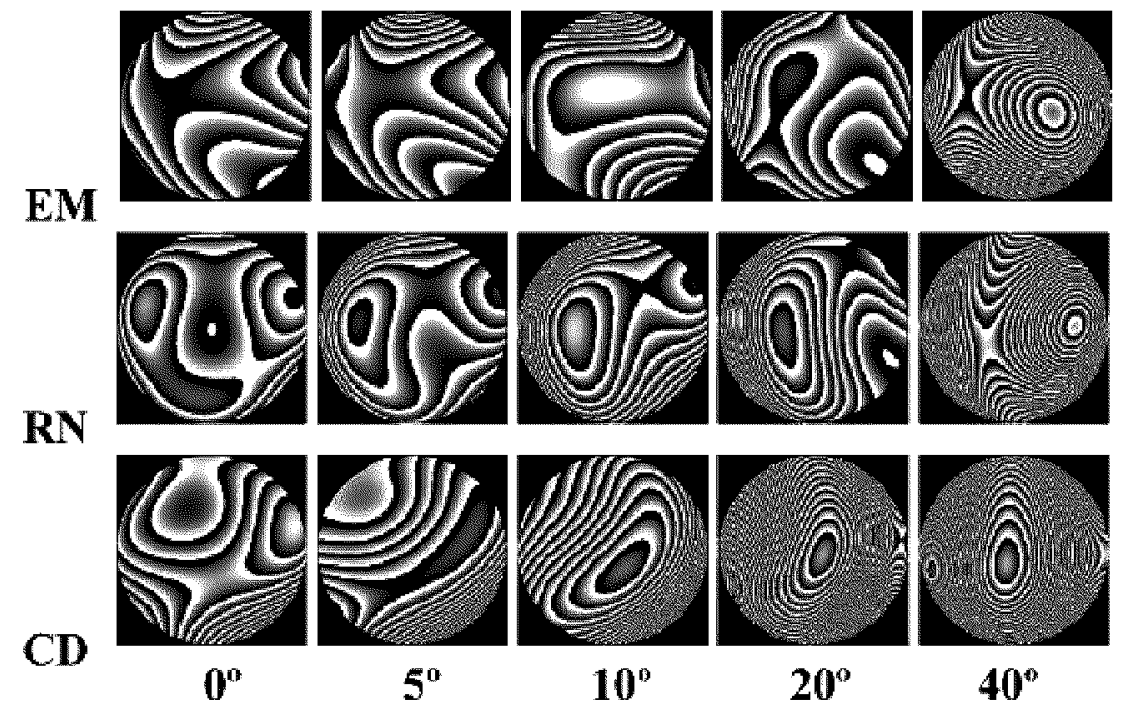

Fig. 4. Wave aberration across visual field (from 0 to 40 degrees) for 3 subjects, measured by LRT.

In parallel to these experimental studies, schematic eye models, initially designed to reproduce the on-axis performance of the eye, can be generalized to reproduce off-axis performance [17] as well. Interestingly, although these schematic models are far from reproducing individual eyes, they can help to understand the main features of the optical design of the eye, permitting a global analysis of average experimental data. In this way, it becomes clear, how aberrations, which are 
relatively low for small pupils (daylight conditions), rapidly increase with a pupil size, becoming large during the night. Or how the relatively good off-axis quality is explained by the fact that the combined effect of off-axis (oblique) astigmatism and field curvature makes that the locus of the best image (the image surface) is in close match with the retinal surface (roughly spherical). We could conclude that the eye has been designed as a very wide angle lens, having a moderately stable optical performance across visual field.

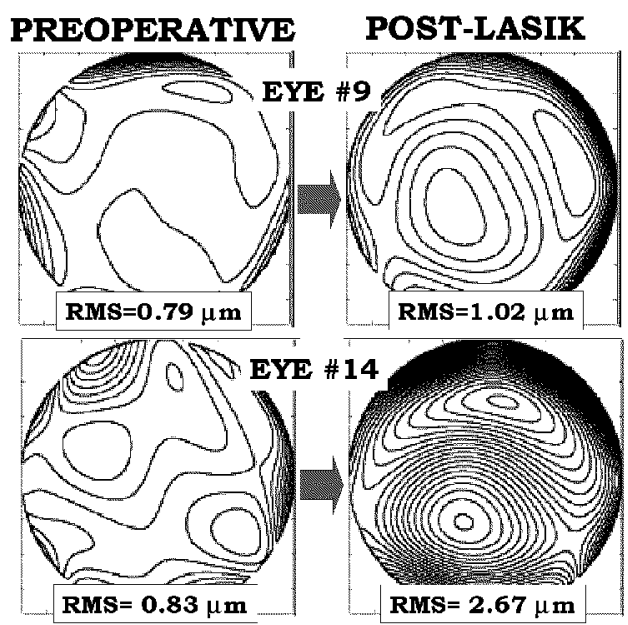

Fig. 5. Best-corrected (canceling defocus and astigmatism) wave aberration error for 2 eyes (\#9 and \#14) before and after LASIK refractive surgery. Values of the RMS wavefront error are also included.

As an example of clinical application, the effect of LASIK refractive surgery upon the optical quality of the eye has been studied, comparing the wave aberration before and after surgery in the same eyes [8]. Figure 5 shows the results for two representative examples. For eye number 9 (upper row) aberrations increase moderately, about $30 \%$, whereas for eye number 14 (lower row) the aberrations increase by a factor of 3 . The results of that study show that the overall aberrations (excluding first and second order ones) increase by a factor of 2 on average. The main contribution to the increase in aberration comes from spherical aberration, which is in agreement with previous findings. Coma-like (3rd order) aberrations have a higher contribution than 4 th order ones even in postoperative eyes, although this seems to be strongly dependent on the degree of decentration of the ablation pattern. Fifth and higher order aberrations are not much affected by surgery. Due to the increase in ocular aberrations after LASIK, the average MTF decreases for all spatial frequencies. Even for small pupils $(3 \mathrm{~mm})$ the optical quality of operated eyes is significantly worse than for preoperative eyes, but the aberration increment is considerably smaller than for scotopic pupil sizes $(6.5 \mathrm{~mm})$. 


\section{Aberration compensation}

There have been different experiments demonstrating the feasibility of correcting the aberrations of the eye. In all cases, the method consists of two stages: (1) measurement and (2) compensation. The compensation is achieved by some optical system (deformable mirror, phase plate, etc.) which transforms a perfect (flat) incoming wavefront into an "aberrated" one, that is conjugate to the eye's wave aberration, so that the joint system (eye + correcting device) has no aberrations and works as a perfect optical system. The process can be dynamic introducing a control loop, so that the system adapts to potential temporal changes of aberrations (adaptive optics). The compensation can be static, such as is the case of ophthalmic lenses (spectacles or contact), or they could be induced permanently by modifying the optical system of the eye by refractive surgery. Whereas there are serious experimental results in the two first cases, surgery has not provided convincing results yet, in spite of new commercially available surgery devices guided by ocular aberrations.

\subsection{Adaptive optics}

The first adaptive optics system for the eye was developed at the University of Rochester [9]. It consisted of a Hartmann-Shack wavefront sensor, plus a deformable mirror with 37 actuators, both connected by a control loop. Figure 6

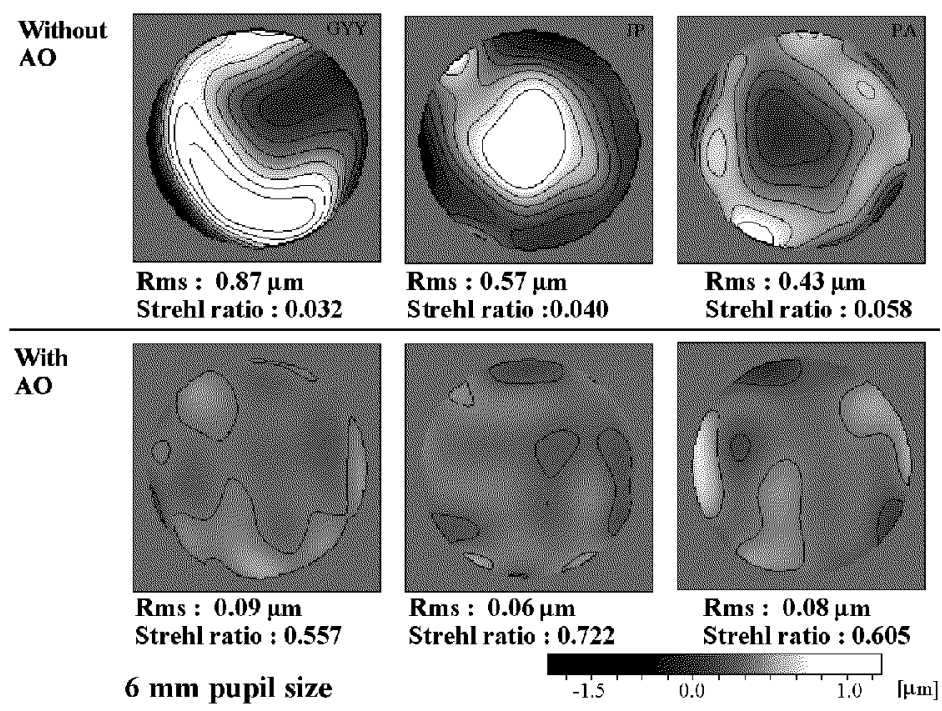

Fig. 6. Wavefront aberration measured with the Rochester adaptive optics system OFF (upper row) and $\mathrm{ON}$ (lower row). With the AO system ON, the Strehl ratios become much closer to the diffraction limit $(0.8)$ (by courtesy of D.R. Williams). 
shows recent results obtained with that system. When the adaptive optics system is $\mathrm{ON}$, then the aberrations are compensated. In some cases, they are reduced by a factor of almost 10 , and the resulting optical quality is almost diffraction limited. This system has permitted to improve the optical resolution of fundus cameras, allowing to resolve single retinal cells, such as cone photoreceptors. In this way they could perform totally new experiments, as for instance, to obtain the arrangement of the three cone classes in the living human eye [18]. The last version of this system works in real time. Correction of the eye's aberrations can be completed in $0.25-0.5$ seconds, resulting in residual RMS wavefront errors as low as 0.1 microns for $6.8 \mathrm{~mm}$ pupils [19].

\subsection{Phase plates}

Adaptive optics devices can probably provide the best possible results. However they are highly sophisticated, and they could hardly go outside the optical laboratory or clinic. The idea of using phase plates is to produce a small and light optical element to work in the same way as contact or spectacle lenses, providing a static correction. The manufacture process consists of a gray-level single-mask photosculpture in photoresist technique, which is a reasonably efficient and cheap method for making microoptics elements. After passing optical tests (Fig. 7), the plate is placed in front of the eye, like a spectacle lens and we measure the wave aberration again to evaluate the degree of compensation attained, as a global assessment of the whole procedure [10].
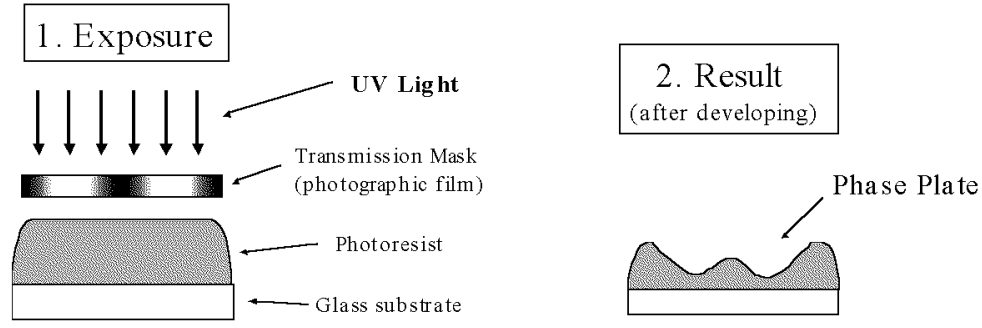

Fig. 7. Phase plate manufacturing process. The photoresist layer, spin-coated onto a glass substrate, is exposed to UV light through a gray-level transmission mask, so that a continuous profile of photoresist thickness is obtained after development. These thickness differences induce phase shifts on the incident beam.

Results for one subject are shown in Fig. 8. The initial (original) wave aberration (left) is compensated by means of the phase plate (middle). The resulting overall wave aberration of the eye + plate system (right) is about 5 times lower. This result looks promising since further improvements of the method are still possible (especially in the plate manufacturing stage). In fact here we reached the maximum theoretical or potential compensation attainable by this plate. By 
Original W. A.

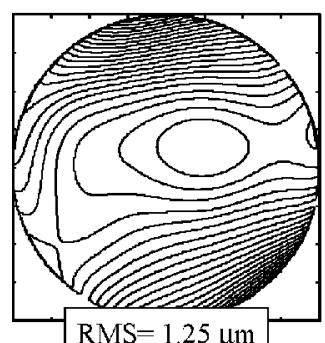

RMS $=1.25 \mu \mathrm{m}$
Plate Interferogram

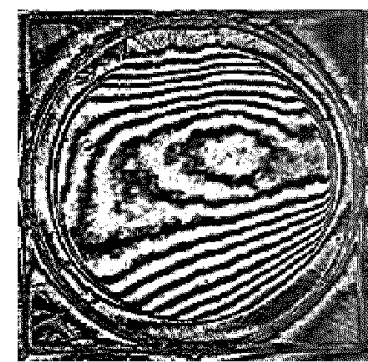

After correction

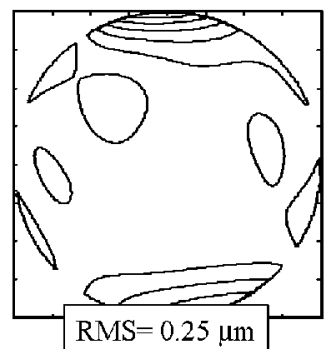

Fig. 8. Wave aberration contour plot for subject EM, right eye, before (left) and after (right) compensation with the customized phase plate (middle), placed in front of the eye in the manner of a spectacle lens. Step between adjacent contour lines is $0.5 \mu \mathrm{m}$.

measuring and adding eye's and plate's wave aberrations, the estimated minimum residual aberration was $0.25 \mu \mathrm{m}$, that is the value found experimentally. This suggests that improvements in the result (higher degrees of compensation) are still possible by optimizing the manufacturing process to obtain plates with a higher fidelity. One of the major limitations of the methods to correct aberrations is that the alignment is critical. The alignment of conventional lenses to correct second order aberrations is not critical at all, but as the order of the aberrations to be corrected increases, the more critical is the alignment [20]. Therefore, in most practical applications, phase plates or similar elements will be limited to correct up to 4th order aberrations (coma, spherical, etc.).

\section{Conclusions and future trends}

In conclusion there are accurate and robust methods to measure ocular aberrations. The high degree of compensation attained in different experiments in different laboratories confirms the accuracy and reliability of the measurements. This has permitted to assess the optical quality of the human eye better than ever before. As a consequence, the amount of experimental results in both basic and clinical studies is growing very rapidly, as well as our knowledge of the optics of the eye. The correction of aberration has been also demonstrated in human eyes. This opens new fields of research and applications, not only to achieve a supernatural vision, but also the possibility of resolving retinal cells, such as the photoreceptors is making possible new clinical applications as well as new experiments, stimulating single cones, etc.

This field of research is relatively new although the number of laboratories is growing rapidly because there are many open questions, and many technologies have still to be developed. An important effort is being made to make fast measurements to study the dynamics of aberrations and achieve real time instantaneous correction. Measurements in the near infrared are especially interesting 
in many applications, but then it is important to know to what extent one can extrapolate these data to the visible spectrum. A crucial stage for the future development of refractive surgery methods is to develop realistic customized eye models able to predict accurately not only the global aberrations of individual eyes, but also of their main elements, cornea and lens. Regarding adaptive optics (AO), there are today two opposite tendencies: on the one hand, to increase the degree of sophistication using faster and better devices with the goal of obtaining true diffraction-limited optical performance. On the other hand, several laboratories are developing cheaper systems affordable for basic and clinical laboratories. However, the most important part is to find new applications of AO systems. In static correction it is crucial to improve the manufacture techniques and develop customized spectacle lenses, contact lenses, eye pieces, etc. In real applications, it will be also important to achieve a wide field compensation, which will make necessary to optimize the designs of the correcting optical elements. Finally, we have to expect that refractive surgery techniques used to correct 2 nd order aberrations, but which are now inducing $3 \mathrm{rd}$ and 4 th order aberrations, will be soon improved so that they do not increase aberrations but even correct them.

\section{Acknowledgments}

Thanks to David R. Williams for providing figures and to my main collaborators in the different aspects of this line of research: Esther Moreno and Susana Marcos from the Instituto de Óptica and Salvador Bará and Teresa Mancebo from the Área de Óptica, Universidade de Santiago de Compostela.

\section{References}

[1] H.C. Howland, B. Howland, J. Opt. Soc. Am. A 67, 1508 (1977).

[2] J. Liang, B. Grimm, S. Goelz, J. Bille, J. Opt. Soc. Am. A 11, 1949 (1994).

[3] R. Navarro, M.A. Losada, Optom. Vision Sci. 74, 540 (1997).

[4] P. Mierdel, H.E. Krinke, W. Wiegand, M. Kaemmerer, T. Seiler, Ophthalmologe 94, 441 (1997).

[5] J.C. He, S. Marcos, R.H. Webb, S.A. Burns, J. Opt. Soc. Am. A 15, 2449 (1998).

[6] T. Oshika, S.D. Klyce, R.A. Applegate, H.C. Howland, M.A. El Danasoury, Am. J. Ophthalmol. 127, 1 (1999).

[7] T. Seiler, M. Kaemmerer, P. Mierdel, H.-E. Krinke, Arch. Ophthalmol. 118, 17 (2000).

[8] E. Moreno-Barriuso, J. Merayo-Lloves, S. Marcos, R. Navarro, L. Llorente, S. Barbero, Invest. Ophthalmol. Vis. Sci. 42, 1396 (2001).

[9] J. Liang, D.R. Williams, D.T. Miller, J. Opt. Soc. Am. A 14, 2884 (1997).

[10] R. Navarro, E. Moreno-Barriuso, S. Bará, T. Mancebo, Opt. Lett. 25, 236 (2000).

[11] M.S. Smirnov, Biofizika 6, 687 (1961). 
[12] G. Walsh, W.N. Charman, H.C. Howland, J. Opt. Soc. Am. A 1, 987 (1984).

[13] E. Moreno-Barriuso, R. Navarro, J. Opt. Soc. Am. A 17, 974 (2000).

[14] D. Malacara, Optical Shop Testing, 2nd Ed., John Wiley \&Sons, New York 1992.

[15] J. Porter, A. Guirao, I.G. Cox, D.R. Williams, J. Opt. Soc. Am. A 18, 1793 (2001).

[16] R. Navarro, E. Moreno, C. Dorronsoro, J. Opt. Soc. Am. A 15, 2522 (1998).

[17] I. Escudero-Sanz, R. Navarro, J. Opt. Soc. Am. A 16, 1 (1999).

[18] A. Roorda, D.R. Williams, Nature 397, 520 (1999).

[19] H. Hofer, L. Chen, G.Y. Yoon, B. Singer, Y. Yamauchi, D.R. Williams, Optics Express 8, 631 (2001).

[20] S. Bará, T. Mancebo, E. Moreno-Barriuso, Appl. Opt. 39, 3413 (2000). 